\title{
Wirksamkeit der schulischen Vor- und Nachbereitung eines Schülerlaborbesuches
}

\author{
Maria Reimann $^{1}$ (D) - Stefanie Herzog ${ }^{1}$ (D) - Ilka Parchmann ${ }^{1} \cdot$ Stefan Schwarzer $^{2}$ (D
}

Eingegangen: 22. April 2020 / Angenommen: 7. Oktober 2020 / Online publiziert: 2. November 2020

(c) Der/die Autor(en) 2020

\section{Zusammenfassung}

MINT-Schülerlabore sind non-formelle Lernorte, welche den Schulunterricht ergänzen sollen. Aufgrund ihrer anschaulichen, interaktiven und motivierenden Darbietungsmethoden können sich positive Effekte auf psychologische und kognitive Konstrukte zeigen, welche durch eine Verknüpfung mit dem schulischen Lernort nachhaltig gesichert werden sollen. Hierbei können berufsorientierende, interessebasierte und motivationale Aspekte sowie das Fähigkeitsselbstkonzept, die Selbstwirksamkeit und der Wissenserwerb positiv und langfristig beeinflusst werden. Im Rahmen dieser Studie wurde mit standortübergreifenden Daten von 752 gymnasialen und nicht-gymnasialen Lernenden anhand einer Prä-Post-Follow-up-Erhebung die Entwicklung der beschriebenen Konstrukte durch einen einfachen Schülerlaborbesuch sowie eine zusätzliche rein fachliche bzw. berufsorientierte schulische Vor- und Nachbereitung untersucht. Hierbei konnte durch die Intervention lediglich bei den kognitiven jedoch nicht bei den psychologischen Konstrukten eine positive Änderung erreicht werden. Zudem wurde im Rahmen eines Vergleichs der Konstrukt-Ausprägungen in den beiden Schulformen festgestellt, dass gymnasiale Lernende stärker selbstbestimmte motivationale Regulationen nutzen als nicht-gymnasiale Schülerinnen und Schüler. Diese Ergebnisse müssen aus diversen Blickwinkeln diskutiert werden.

Schlüsselwörter Schülerlabor · Schulische Vor- und Nachbereitung · Berufsorientierung

\section{Effectiveness of Preparation and Follow-up Lessons in School of a Student Lab Visit}

\begin{abstract}
STEM student laboratories are non-formal learning environments that are intended to complement classroom teaching. As they make use of visual, interactive and motivating techniques of presenting the science content, they can have positive effects on psychological and cognitive constructs. By linking these to the classroom through preparatory and follow-up lessons, content knowledge and psychological attitudes should be more sustainable. Aspects regarding vocational orientation and motivation and based on interest as well as the academic self-concept, self-efficacy and knowledge acquisition can be influenced in a positive manner in the long run. Using data from a pre-post-follow-up survey of 752 students from both academic-track schools (Gymnasium) and non-academic track schools (Gesamtschule), this study investigates the development of the above-mentioned constructs after a single student lab visit and complementary preparation and follow-up lessons in school focusing on the science content or also on job-oriented aspects. A positive effect on the cognitive constructs but not on the psychological ones could be observed. In addition, a comparison of the two school types indicated that students from the Gymnasium show higher use of self-determined motivational regulations than the students from the Gesamtschule. A critical evaluation of these results from various perspectives is necessary.
\end{abstract}

Keywords Student laboratories · Preparation and follow-up lessons in school · Vocational orientation

Maria Reimann

reimann@ipn.uni-kiel.de

1 IPN, Didaktik der Chemie, Universität Kiel, Olshausenstr. 62, 24118 Kiel, Deutschland
2 Didaktik der Chemie, Eberhard Karls Universität Tübingen, Auf der Morgenstelle 18, 72076 Tübingen, Deutschland 


\section{Einleitung}

Die Förderung des Kompetenzbereichs Erkenntnisgewinnung von Schülerinnen und Schülern soll zur Ausbildung ihrer naturwissenschaftlichen Grundbildung beitragen und findet nicht ausschließlich in der Schule sowie bei schulischen Veranstaltungen, sondern ebenso an außerschulischen Institutionen, wie etwa in einem Schülerlabor, statt (Brandt 2005; Stocklmayer et al. 2010).

Durch die damit einhergehenden Einblicke in naturwissenschaftliche Facetten gesellschaftlicher Prozesse und späterer Berufsfelder erlangen Lernende Vorstellungen über die Rolle und Bedeutung der Naturwissenschaften in ihrer natürlichen, kulturellen und persönlichen Umwelt. Damit sollen sie in die Lage versetzt werden, sich eine eigene, begründete Meinung zu bilden, an politischen und wissenschaftlichen Diskussionen teilzuhaben, sich an der Optimierung und dem Fortschritt der Gesellschaft zu beteiligen (Burmeister et al. 2012) und berufliche Wahlentscheidungen zu treffen. Dies kann in der Schule allein nicht ausreichend geleistet werden, weshalb die Bedeutung informeller und non-formeller Lernorte in den letzten 20 Jahren für die MINT-Fächer (Mathematik, Informatik, Naturwissenschaften, Technik) stark zugenommen hat. Der Nachweis nachhaltiger Wirkungen, insbesondere auf die spätere Berufswahlentscheidung, steht bislang jedoch weitestgehend aus (Nickolaus et al. 2018). Diesbezüglich wird eine Verknüpfung schulischer und außerschulischer Lernorte von Lernenden positiv eingeschätzt, etwa durch die Verbindung zusätzlicher Erfahrungen mit der schulischen Grundbildung sowie der Erweiterung von Lernchancen (Itzek-Greulich und Vollmer 2017). Ziel des Projekts, das der hier vorgestellten Studie zugrunde lag, war es daher, die Wirksamkeit einer schulischen Vor- und Nachbereitung eines Schülerlaborbesuchs hinsichtlich psychologischer und kognitiver Konstrukte zu untersuchen. Zusätzlich wurde anhand zweier Varianten der schulischen Vor- und Nachbereitung - mit sowie ohne integrierte Aspekte zur Berufsorientierung der Einfluss berufsorientierender Intervention auf die Einstellung zu MINT-Berufen analysiert. Während der inhaltliche Aufbau des Laborprogramms sowie der unterrichtlichen Vor- und Nachbereitung ausführlich an anderer Stelle beschrieben ist (Reimann et al. 2020), geht es in diesem Artikel darum, zu prüfen, inwieweit verschiedene Aspekte als Stellschrauben genutzt werden können, um bei Lernenden die Motivation zu wecken, sich mit naturwissenschaftlichen Inhalten zu beschäftigen - auch im Hinblick auf eine naturwissenschaftsbezogene Berufsorientierung.

\section{Theoretischer Hintergrund}

Im Rahmen des Projekts wurden begleitend zur Durchführung des Schülerlabortags sowie der Vor- und Nachbereitung mit dem Thema Nanotechnologie verschiedene Aspekte erhoben, die in diesem Beitrag kurz im Zusammenhang zu Motivation und Lernen in den Naturwissenschaften vorgestellt, ausgewertet und als möglicherweise auch für andere Projekte zielführend präsentiert werden. Diese Aspekte umfassen sowohl berufsorientierende als auch motivationale Konstrukte sowie das Fähigkeitsselbstkonzept, die Selbstwirksamkeit und den Wissenserwerb.

Bei der Berufswahl handelt es sich um eine Interaktion zwischen persönlichen und kontextuellen Variablen, die sich in den sozial-kognitiven Mechanismen Selbstwirksamkeit, Ergebniserwartung und Zielvorstellung äußern (Sasson 2019). Nach wie vor ist weltweit in bestimmten Bereichen ein Mangel an naturwissenschaftlich interessierten und orientierten Schülerinnen und Schülern zu verzeichnen (Elster 2009). Zudem lässt sich feststellen, dass MINT-Berufe weiterhin von einem negativen Image begleitet werden und Lernende oftmals unklare Vorstellungen zu diesen Berufen aufweisen (Elster 2009; Weßnigk 2013). Ein Grund dafür kann die nicht zureichende Gestaltung berufsorientierender Maßnahmen durch Lehrkräfte im MINT-Unterricht sein, da diese selbst vielfach nicht über ausreichende Kenntnisse und Einblicke in heutige Berufsfelder verfügen (Haucke 2013). Die Wirksamkeit und Umsetzung von Maßnahmen ist dabei ebenfalls von der Schulform abhängig (Wirth 2019). Studien zur Förderung einer weiterführenden MINT-Orientierung weisen darauf hin, dass frühe Einblicke in naturwissenschaftliche Berufsfelder zu höherem Interesse und Motivation in naturwissenschaftlichen Bereichen führen (Reiss und Mujtaba 2017). Somit ist ein frühes, schülergerechtes und anschaulich gestaltetes Angebot an Methoden, Verfahren und Denkweisen sinnvoll, wie es in Forschung und Technologieentwicklung sowie in handwerklichen Berufen angewandt wird.

Die Lernmotivation lässt sich gemäß der Selbstbestimmungstheorie von Ryan und Deci (2000) in intrinsische und extrinsische Regulationsstile aufteilen. Die intrinsische Motivation ist vollkommen selbstbestimmt (Ryan und Deci 2000) und hängt eng mit der Berufsorientierung zusammen (Taskinen et al. 2013). Die extrinsische Motivation, deren Ziel außerhalb der Handlung liegt, kann in vier Regulationsstile - externale, introjizierte, identifizierte und integrierte unterteilt werden, wobei deren Handlungsqualität von heteronomer Kontrolle bis zur Selbstbestimmung reicht (Ryan und Deci 2000). Dabei ist der integrierte Regulationsstil kaum mehr von der intrinsischen Motivation zu unterscheiden (Ryan und Deci 2000). Intrinsische und extrinsische Regulationen können bei einem Lernenden ebenfalls nebeneinander existieren und zu einer kumulierten Lernmoti- 
vation führen (Müller et al. 2007). Studien im Bereich der Naturwissenschaften konnten bereits mehrfach zeigen, dass selbstbestimmtes Lernen sowie eigenständiges Experimentieren und Erarbeiten von Inhalten zu positiven Auswirkungen und Förderung der intrinsischen Motivation führen kann, was sich oftmals in besseren Lernleistungen äußert (Weßnigk 2013; Euler 2005; Grolnick et al. 1991). Strikte Anleitungen führen hingegen kaum zu positiven Effekten (Euler 2005).

Das Fähigkeitsselbstkonzept stellt einen weiteren Prädiktor für die Berufsorientierung dar (Baumert 1992), welches auf der fachbezogenen Einschätzung der eigenen Fähigkeiten und Fertigkeiten in akademischen Leistungssituationen (Dickhäuser 2006) basiert und eine Komponente des allgemeinen Selbstkonzepts ist. Einige Studien konnten bereits zeigen, dass das Fähigkeitsselbstkonzept bedeutsam für spätere Lernleistungen ist (Köller et al. 2006) und sich auf das Niveau und die Komplexität der angestrebten Laufbahn auswirkt (Filipp 2006). Das allgemeine Selbstkonzept ist ein stabiles psychologisches Konstrukt, wohingegen das Fähigkeitsselbstkonzept unter bestimmten Bedingungen über die Zeit beeinflusst werden kann. So können vor allem motivierende und erfolgsbasierte Lernsituationen, welche stärker lernziel- und weniger leistungszielorientiert sind, eine positive Beeinflussung des naturwissenschaftlichen Fähigkeitsselbstkonzepts hervorrufen (Pawek 2009; Weßnigk 2013).

Das kognitive Konstrukt Selbstwirksamkeit hängt stark mit der Bewusstseinsbildung zusammen und äußert sich im Verhalten in Lern- und Leistungssituationen (Bandura 1997). Hierbei handelt es sich um die subjektive Wahrnehmung der eigenen Kompetenzen, wobei sich mit steigender Selbstwirksamkeit auch die Motivation zur Bewältigung von anspruchsvolleren Situationen und Aufgaben erhöht (Schwarzer und Jerusalem 2002). Dabei fördern eigenverantwortliche und kontextorientierte Arbeitsweisen, wie diese oft an außerschulischen Lernorten gegeben sind, eine positive Entwicklung der Selbstwirksamkeit (Haupt 2018).

Ebenfalls beim Fachwissen und der damit verbundenen Lernleistung handelt es sich um ein kognitives Konstrukt, welches zum einen von Schülerfaktoren wie der aufgabenspezifischen Begabung, der Annahmefähigkeit einer Lerngelegenheit sowie der Ausdauer und zum anderen von äuBeren Bedingungen wie der vorgesehenen Lernzeit sowie der Qualität der Darbietungsmethode abhängt (Rheinberg 1996). So zeigen diverse Studien (Wilde und Bätz 2006; Guderian 2007; Itzek-Greulich 2014; Klees und Tillmann 2015), dass Schülerinnen und Schüler an einem außerschulischen Lernort besser und effektiver lernen und die gelernten Inhalte nachhaltiger behalten können, wenn sie vorab auf den Besuch vorbereitet wurden.

\section{Auswirkungen eines Schülerlaborbesuchs}

Schülerlabore sind ein besonderes Format non-formeller Lernorte, welche vor allem in Deutschland mit mittlerweile etwa 380 Institutionen (LernortLabor 2020) sehr weit verbreitet sind, aber auch in anderen Ländern, wie etwa Israel und Finnland, Anwendung finden. Zahlenmäßig sind MINT-Schülerlabore mit Abstand am häufigsten vertreten. Viele dieser Labore setzen sich das Ziel, Einblicke in Forschung zu ermöglichen und somit Wissenschaftskommunikation zu praktizieren (LernortLabor 2017). Diese erlauben ein handlungsorientiertes Arbeiten mit, im Vergleich zum Schulunterricht, mehr Zeit für Eigenaktivität und Schülerexperimente (Euler und Weßnigk 2011) zu aktuellen naturwissenschaftlichen Themen in einer authentischen Umgebung (Braund und Reiss 2006).

Außerschulische Lernorte können durch ihre häufig interaktiven Darbietungsmethoden der Lerninhalte sowie die Schaffung einer erfolgsorientierten und herausfordernden Lernsituation ohne vorhandenen Leistungsdruck bei den Lernenden (situatives) Interesse und Motivation stärken (Braund und Reiss 2006; Nickolaus et al. 2018) sowie ein verbessertes Fähigkeitsselbstkonzept (Weßnigk 2013; Wirth 2019) und eine höhere Selbstwirksamkeit (Haupt 2018) hervorrufen. Zudem können diese einen starken Einfluss auf das Interesse an MINT-Berufen haben (Weßnigk 2013; Haucke 2013), da hier Erfahrungen gemacht werden können, die über den normalen Alltag (Schule, soziale Umgebung) hinausgehen.

Wird jedoch die Nachhaltigkeit einer derartigen nonformellen Institution betrachtet, so lässt sich oftmals lediglich eine kurz- bis mittelfristige Stabilisierung der positiven Effekte feststellen (Schwarzer und Itzek-Greulich 2015). Eine Möglichkeit zur Lösung dieses Problems wäre eine Verknüpfung zwischen schulischen und außerschulischen Lernorten (Brandt 2005). Diese trägt insgesamt zur Erhöhung der Produktivität und Nachhaltigkeit eines Schülerlaborbesuchs bei (Schwarzer und Itzek-Greulich 2015). Zum einen schafft dies eine wiederholte Konfrontation mit den dargebotenen Inhalten, wodurch bereits erste Einblicke in die Thematik gegeben, das Verständnis der Inhalte erleichtert, die fachlichen Diskussionen unterstützt und vertieft sowie anschließend gelernte Inhalte gesichert werden können. Zum anderen erlangt der Laborbesuch bei den Schülerinnen und Schülern einen höheren Stellenwert, da dieser mit dem Unterricht gekoppelt ist und somit offensichtlich eine Relevanz für die Schule, den Unterricht sowie den Lern- und Leistungsprozess hat (Wilde und Bätz 2006; Klees und Tillmann 2015). Ebenso können dadurch die Novelty Space Effekte verringert werden (Orion und Hofstein 1994). Studien konnten zeigen, dass die Lernfähigkeit und Selbstwirksamkeit sowie die Lernleistung von Schülerinnen und Schülern stark mit der Vertrautheit der Lernumgebung 
zusammenhängen (Falk 1983; Falk et al. 1978). Hier spielen die Erfahrung mit außerschulischen Lernorten sowie das vorhandene Vorwissen bzgl. der thematischen Ausrichtung der Institution eine wichtige Rolle (Orion und Hofstein 1994). Findet im Vorhinein keine Auseinandersetzung mit den Merkmalen des Lernortes statt, so wird dieser Prozess von den Lernenden selber zunächst in der neuen Lernumgebung nachgeholt, bevor eine kognitive Auseinandersetzung mit den Lerninhalten erfolgen kann (Orion und Hofstein 1994). Somit ist zur Steigerung der pädagogischen Effektivität eine Verringerung der Novelty Space Effekte sinnvoll. Eine Möglichkeit einer derartigen Verknüpfung von Lernorten ist eine thematische und auf den außerschulischen Lernort bezogene schulische Vor- und Nachbereitung (Schwarzer und Itzek-Greulich 2015).

In Schülerlaboren mit dem Fokus Berufsorientierung kann im Zusammenspiel schulischer und außerschulischer Fördermaßnahmen das Ziel der Nachwuchsförderung durch Steigerung von intrinsischer Motivation, dem Fähigkeitsselbstkonzept und Interesse im naturwissenschaftlichen und technischen Bereich gezielt adressiert und nachhaltig gestaltet werden (Affeldt et al. 2015; Guderian und Priemer 2008; Weßnigk 2013). Hier werden diese Konstrukte in einen unmittelbaren Zusammenhang mit Berufen und der eigenen potenziellen Berufswahl (Taskinen et al. 2013) bzw. der Präferenz, naturwissenschaftliche Fachrichtungen weiter zu erlernen, gestellt (Wirth 2019).

Außerschulische Lernorte bieten ihre Programme überwiegend für gymnasiale Lernende an, wobei nicht-gymnasiale Schülerinnen und Schüler weniger außerschulische Förderung erhalten. Studien konnten zudem belegen, dass gymnasiale Lernende unter anderem eine positivere Einstellung zu MINT-Berufen, eine höhere Selbstwirksamkeit, ein besseres Fachwissen sowie ein stärkeres Fähigkeitsselbstkonzept als Lernende nicht-gymnasialer Schulformen zeigen (Wirth 2019). Zudem bedingt die Unterrichtsgestaltung die motivationalen Regulationen (Katz und Assor 2007).

\section{Schülerlaborprogramm nawi:klick!}

Die nachfolgend beschriebene Untersuchung wurde als Begleitforschung des nanotechnologischen Schülerlabors $n a-$ wi:klick! durchgeführt. Dieses Laborprogramm vermittelt Schülerinnen und Schülern gemäß der Definition von Nanotechnologie im Rahmen eines ganztägigen Experimentierangebots die Herstellung, Untersuchung und Anwendung von Nanopartikeln sowie nano- und mikrodimensionierten Oberflächen (Reimann et al. 2020). Hier werden insgesamt sechs fächerübergreifende, interaktive und anschaulich gestaltete Experimentierstationen mit einem eindeutigen Alltagsbezug dargeboten, anhand derer die komplexen nano- technologischen Inhalte schülergerecht und nachvollziehbar erarbeitet werden können.

Das Labor richtet sich an die Klassenstufen 8-10 sowohl gymnasialer als auch nicht-gymnasialer Schulformen. Hierdurch sollen insbesondere Lernende aus Real- und Gemeinschaftsschulen eine Chance erhalten, an einem non-formellen Lernangebot teilzuhaben. Um zudem eine eindeutige Anknüpfung an schulische Lernprozesse schaffen und die Novelty Space Effekte verringern zu können (Orion und Hofstein 1994), wird eine auf das Schülerlaborprogramm abgestimmte schulische Vor- und Nachbereitung mit anteilig integrierten Aspekten der Berufsorientierung angeboten. Entsprechend der Ausrichtung des Lernangebots werden im Rahmen der Berufsorientierung nicht-akademische Ausbildungsberufe fokussiert.

Die schulische Vor- und Nachbereitung wurde in zwei Fassungen - ohne sowie mit integrierten Aspekten der Berufsorientierung - entwickelt. Dadurch soll zum einen für das Schülerlaborprogramm notwendiges Fachwissen erarbeitet, das Verständnis der Inhalte erleichtert, erste Einblicke in die Thematik des Schülerlabors gegeben sowie die fachlichen Diskussionen unterstützt und vertieft werden. Zum anderen können anschließend während des Laborbesuchs erlernte Inhalte gesichert, noch offen gebliebene Fragen geklärt sowie die behandelten Thematiken transferiert und erweitert werden. Die beiden Fassungen der Verknüpfung des formellen und non-formellen Lernortes stimmen bis auf einen Teil, in dessen Rahmen naturwissenschaftliche Berufe erarbeitet werden, inhaltlich überein.

Die Vor- und Nachbereitung findet jeweils in Form einer Doppelstunde an der Schule statt. Dabei werden vor$\mathrm{ab}$ in einer Gruppenarbeit grundlegende nanotechnologische Inhalte eingeführt, welche beim Schülerlabortag an den einzelnen Experimentierstationen vertieft werden. In diesem Rahmen wird zunächst die Definition der Nanotechnologie sowie die Größendimensionen Piko- bis Kilometer erarbeitet und diskutiert. Ebenfalls werden die auf der starken Oberflächenvergrößerung beruhenden außerordentlichen Eigenschaften der Nanomaterialien erörtert und experimentell nachgewiesen. Im Rahmen berufsorientierender Aspekte wird ein Zugang zu naturwissenschaftlichen Ausbildungsberufen geschaffen, welche sich tatsächlich mit Nanotechnologie beschäftigen und mit Nanoprodukten arbeiten könnten. Hierzu diskutieren Lernende diverse Ausbildungsberufe - wie etwa Verfahrensmechaniker/in für Beschichtungstechnik oder pharmazeutisch-technische/r Assistent/in - und deren Anwendungsbereiche. Die Nachbereitung erfolgt zur Reflexion, Wiederholung und Sicherung in Form einer Plakatdarstellung zu den einzelnen Stationen und deren Präsentation im Plenum. Dabei werden in der Gruppe mit Berufsorientierung zusätzlich die bereits aus der Vorbereitung bekannten sowie weitere vorgegebene 
Ausbildungsberufe und lokale Unternehmen den Inhalten der einzelnen Plakate begründet zugeordnet.

\section{Ziel der Studie}

Anknüpfend an den Stand der Forschung fokussiert die im Folgenden dargestellte Intervention die Verknüpfung von schulischem und außerschulischem Lernort, um eine Nachhaltigkeit der positiven Effekte zu fördern. Zudem erfolgt ein Vergleich zwischen gymnasialen und nicht-gymnasialen Schulformen. Die Untersuchung hat folgende Forschungsfragen fokussiert:

FF1 Zeigen sich direkt nach einem Schülerlaborbesuch sowie nach einigen Monaten positive Effekte auf ...

a. ... die Einstellung zu MINT-Berufen, ...

b. ... das Fähigkeitsselbstkonzept, ...

c. ... die motivationale Regulation, ...

d. ... die Selbstwirksamkeit, ...

e. ... das Fachwissen, ...

... wenn der Schülerlaborbesuch durch eine schulische Vor- und Nachbereitung begleitet wird?

FF2 Zeigen sich differentielle Effekte auf die Konstrukte a-e, wenn die Vor- und Nachbereitung neben dem fachlichen Fokus Aspekte der Berufsorientierung aufweist?

FF3 Zeigen sich bei der gymnasialen und nicht-gymnasialen Schulform differentielle Ausprägungen der Konstrukte a-e?

Aus den ersten beiden Forschungsfragen wurden drei Untersuchungsgruppen abgeleitet: die Experimentalgruppe 1 (EG1), welche sowohl einen Schülerlaborbesuch als auch eine rein fachliche schulische Vor- und Nachbereitung durchläuft; die Experimentalgruppe 2 (EG2), bei welcher der Schülerlaborbesuch von einer fachlichen und berufsorientierenden schulischen Vor- und Nachbereitung begleitet wird; sowie die Kontrollgruppe (KG), welche ausschließlich an einem Schülerlabortag teilnimmt. Die dritte Frage konnte untersucht werden, da an dem Programm sowohl gymnasiale als auch nicht-gymnasiale Lerngruppen teilnehmen.

Folgende Hypothesen wurden auf Basis des derzeitigen Stands der Forschung zugrunde gelegt:

H1 Zu allen Messzeitpunkten (MZP) werden die Einstellung zu MINT-Berufen, das Fähigkeitsselbstkonzept, die Selbstwirksamkeit und das Fachwissen bei der gymnasialen Substichprobe positiver bzw. höher ausgeprägt sein als bei der nicht-gymnasialen Substichprobe.
Weitere Hypothesen wurden jeweils für die beiden Schulformen überprüft:

H2 Die Einstellung zu MINT-Berufen wird bei EG2 im Post- und Follow-up-Test positiver sein als im Prätest und bei EG1 sowie KG wird sich keine Änderung gegenüber dem Prätest zeigen.

H3 Das Fachwissen wird vom Post- zum Follow-up-Test bei den beiden Experimentalgruppen stabil bleiben und bei KG sinken.

H4 Das Fähigkeitsselbstkonzept (H4a) und die intrinsische Motivation (H4b) werden bei allen Untersuchungsgruppen im Posttest und bei den beiden Experimentalgruppen ebenfalls im Follow-up-Test positiver bzw. höher sein als im Prätest.

H5 Die Selbstwirksamkeit wird bei allen Untersuchungsgruppen im Post- und Follow-up-Test höher sein als im Prätest.

\section{Methode}

\section{Stichprobe}

Die Untersuchung wurde bewusst in den zwei Bundesländern Schleswig-Holstein in der Kieler Forschungswerkstatt und Bayern an der LMU München durchgeführt, um die Abhängigkeit der Ergebnisse von einem spezifischen Lehrplan oder einer Schulstruktur zu vermeiden. Die Wahl der Bundesländer erfolgte aus organisatorischen Gründen, d.h. hinsichtlich der prinzipiellen Durchführbarkeit der Schülerlabortage am Standort. Aufgrund einer zeitversetzten Anmeldung zum Projekttag konnten die 1605 Schülerinnen und Schüler, die insgesamt am Schülerlabortag teilgenommen haben, nicht zufällig auf die drei Bedingungen verteilt werden. Von $38,4 \%$ der nicht-gymnasialen und 52,4\% der gymnasialen Lernenden liegen vollständige Datensätze $\mathrm{zu}$ allen fünf erhobenen Konstrukten FF1a-e und allen MZP vor, so dass an den beiden Standorten der Studie 752 Schülerinnen und Schüler von 23 Gymnasien $(n=507)$ und 9 nicht-gymnasialen Schulformen $(n=245)$ das Schülerlaborprogramm durchlaufen und durchgehend an der Erhebung teilgenommen haben. Hierbei waren Mädchen $(43,9 \%)$ und Jungen $(56,1 \%)$ aus den Klassenstufen 8 $(n=318), 9(n=285)$ und $10(n=149)$ mit einem Durchschnittsalter von 14,4 Jahren $(S D=1,0)$ beteiligt, von denen $79,3 \%$ das Abitur als Schulabschluss anstreben. Im Hinblick auf die Verteilung der Lernenden in diesem Datensatz auf die beiden Bundesländer haben ca. $45 \%$ der Teilneh- 
menden an dem Schülerlaborprogramm in Bayern und $55 \%$ in Schleswig-Holstein teilgenommen.

\section{Messinstrumente}

Zur Untersuchung der Forschungsfragen wurde eine quantitative fragebogenbasierte Prä-Post-Follow-up-Erhebung der Konstrukte FF1a-e unter Verwendung 4-stufiger Likert-Skalen im quasi-experimentellen Design durchgeführt. Die Fragebögen wurden jeweils in der Schule vor der Vorbereitung $\left(\mathrm{t}_{0}\right)$ und nach der Nachbereitung $\left(\mathrm{t}_{1}\right)$ sowie 12 Wochen später $\left(t_{2}\right)$ eingesetzt. Es konnte in der gymnasialen Substichprobe eine gleichmäßige Aufteilung auf die drei Untersuchungsgruppen erfolgen, wohingegen in der nicht-gymnasialen Substichprobe der Anteil der Probanden in EG1 (43,3\%) gegenüber den in EG2 (25,3\%) und KG $(31,4 \%)$ überwiegt.

Im Rahmen der Befragungen wurden zu allen drei MZP die fünf Konstrukte FF1a-e erhoben und die Reliabilitäten der einzelnen Skalen anhand einer Konsistenzanalyse unter Angabe des Kennwerts Cronbachs Alpha $\left(\alpha_{0}, \alpha_{1}, \alpha_{2}\right)$ schulformübergreifend berechnet (Tab. 1).

Für alle Konstrukte mit Ausnahme des Fachwissens können die Skalenreliabilitäten mit Werten zwischen 0,71 und 0,92 als wenigstens zufriedenstellend bewertet werden. Die Items zu dem Konstrukt Fachwissen stellen im Prätest keine einheitliche Skala dar. Da laut Befragungen die Probanden vorab kein spezifisches Wissen zu nanowissenschaftlichen Thematiken hatten, ist die niedrige Reliabilität vermutlich auf unstrukturiertes Raten, eine Unkenntnis von spezifischem Fachvokabular aber auch erste, richtig erkannte $\mathrm{Zu}$ sammenhänge im Bereich der Nanotechnologie zurückzuführen, die erst im weiteren Verlauf der Intervention vertieft wurden. Somit werden bei den folgenden Auswertungen die Ergebnisse des Konstrukts Fachwissen im Prätest nicht mit einbezogen und die Hypothese $\mathrm{H} 1$ bezogen auf $\mathrm{t}_{0}$ kann aufgrund der beeinträchtigten Testgüte nicht weiterverfolgt werden. Bei der Fragestellung zur Entwicklung wird stattdessen das Fachwissen im Posttest als Start-Wissensausprä-

Tab. 1 Zusammenfassung der erhobenen Konstrukte mit Beispiel-Items, der jeweiligen Anzahl der Items, der Reliabilitäten sowie der eingesetzten Likert-Skala

\begin{tabular}{|c|c|c|c|c|}
\hline Konstrukt & Beispiel-Item & Likert-Skala - numerisch zugeordneter Wert & $\begin{array}{l}\text { Anzahl } \\
\text { Items }\end{array}$ & Reliabilitäten \\
\hline $\begin{array}{l}\text { Einstellung zu } \\
\text { MINT-Berufen } \\
\text { (Weßnigk 2013) }\end{array}$ & $\begin{array}{l}\text { Ich könnte mir vorstellen, eine } \\
\text { Berufsausbildung im } \\
\text { naturwissenschaftlichen Bereich } \\
\text { zu machen }\end{array}$ & $\begin{array}{l}\text { Stimmt völlig }-4 \text {; } \\
\text { stimmt eher }-3 ; \\
\text { stimmt kaum }-2 ; \\
\text { stimmt gar nicht }-1\end{array}$ & 6 & $\begin{array}{l}\alpha_{0}=0,77 \\
\alpha_{1}=0,80 \\
\alpha_{2}=0,78\end{array}$ \\
\hline $\begin{array}{l}\text { Fähigkeitsselbstkonzept } \\
\text { (Pawek 2009) }\end{array}$ & $\begin{array}{l}\text { Obwohl ich mir Mühe gebe, fallen } \\
\text { mir Naturwissenschaften schwer }\end{array}$ & & 6 & $\begin{array}{l}\alpha_{0}=0,84 \\
\alpha_{1}=0,87 \\
\alpha_{2}=0,85\end{array}$ \\
\hline $\begin{array}{l}\text { Motivation } \\
\text { (Müller et al. 2007) }\end{array}$ & $\begin{array}{l}\text { Ich lerne Naturwissenschaften, } \\
\text { weil ... }\end{array}$ & & & \\
\hline Intrinsisch & ... ich neue Dinge lernen möchte & & 5 & $\begin{array}{l}\alpha_{0}=0,88 \\
\alpha_{1}=0,91 \\
\alpha_{2}=0,91\end{array}$ \\
\hline Identifiziert & $\begin{array}{l}\text {... ich damit mehr Möglichkeiten } \\
\text { bei der späteren Berufswahl habe }\end{array}$ & & 4 & $\begin{array}{l}\alpha_{0}=0,83 \\
\alpha_{1}=0,82 \\
\alpha_{2}=0,82\end{array}$ \\
\hline Introjiziert & $\begin{array}{l}\text {... ich ein schlechtes Gewissen } \\
\text { hätte, wenn ich wenig tun würde }\end{array}$ & & 4 & $\begin{array}{l}\alpha_{0}=0,76 \\
\alpha_{1}=0,78 \\
\alpha_{2}=0,78\end{array}$ \\
\hline Extrinsisch & $\begin{array}{l}\text {... ich sonst Ärger mit meiner } \\
\text { Lehrkraft bekomme }\end{array}$ & & 4 & $\begin{array}{l}\alpha_{0}=0,73 \\
\alpha_{1}=0,74 \\
\alpha_{2}=0,71\end{array}$ \\
\hline $\begin{array}{l}\text { Selbstwirksamkeit } \\
\text { (selbst erstellt; in } \\
\text { Anlehnung an Prenzel } \\
\text { et al. 2008) }\end{array}$ & $\begin{array}{l}\text { Wie einfach wäre es für dich zu } \\
\text { begründen, warum Kandiszucker } \\
\text { sich langsamer in Wasser löst als } \\
\text { Haushalts- oder Puderzucker? }\end{array}$ & $\begin{array}{l}\text { Das wäre einfach für mich }-4 \text {; } \\
\text { mit etwas Mühe wäre das machbar für mich } \\
-3 \text {; } \\
\text { es wäre für mich alleine schwer zu lösen }-2 \text {; } \\
\text { das könnte ich nicht }-1\end{array}$ & 10 & $\begin{array}{l}\alpha_{0}=0,83 \\
\alpha_{1}=0,82 \\
\alpha_{2}=0,87\end{array}$ \\
\hline $\begin{array}{l}\text { Fachwissen } \\
\text { (selbst erstellt) }\end{array}$ & $\begin{array}{l}\text { Sind nano-große Teilchen meist } \\
\text { reaktiver als größere Teilchen } \\
\text { desselben Stoffs? }\end{array}$ & $\begin{array}{l}\text { Multiple-choice Wissenstests mit je } 4 \\
\text { verschiedenen Antwortmöglichkeiten }\end{array}$ & 11 & $\begin{array}{l}\alpha_{0}=0,39 \\
\alpha_{1}=0,66 \\
\alpha_{2}=0,67\end{array}$ \\
\hline
\end{tabular}


gung betrachtet, um die Entwicklung zum Follow-up-Test bewerten zu können.

\section{Ergebnisdarstellung}

Die hier beschriebene Studie fokussiert zum einen die Wirksamkeit von schulischer Vor- und Nachbereitung eines Schülerlaborbesuches sowie den Vergleich zwischen gymnasialen und nicht-gymnasialen Schulformen. In der folgenden Ergebnisdarstellung werden somit zum einen die Ausprägungen der Konstrukte der beiden Schulformen miteinander verglichen und zum anderen die Entwicklung der Konstrukte über die MZP innerhalb der einzelnen Schulformen analysiert.

Zur Analyse der einzelnen Konstrukte sowie der Überprüfung der Hypothesen wird jeweils zunächst eine dreifaktorielle Varianzanalyse (ANOVA) mit den Faktoren Schulform (gymnasial vs. nicht-gymnasial) und Untersuchungsgruppen (KG vs. EG1 vs. EG2) durchgeführt. Der dritte Faktor ist der Messwiederholungsfaktor mit drei $\left(t_{0}, t_{1}, t_{2}\right)$ bzw. im Falle des Fachwissens zwei Stufen $\left(t_{1}, t_{2}\right)$. Ausgehend von diesem gemeinsamen Modell unter Verwendung der 752 vollständigen Datensätze werden je nach Hypothe- se jeweils spezifische Werte, die die dreifaktorielle ANOVA liefert, ausgewählt und berichtet.

\section{Vergleich der Konstrukte zwischen den Schulformen zu allen MZP (H1)}

Wird für die einzelnen Konstrukte eine dreifaktorielle ANOVA durchgeführt, so zeigt sich durchgehend der Haupteffekt für Schulform: Einstellung zu MINT-Berufen, $F(1 ; 746)=67.291 ; p<0,001 ; \eta_{\mathrm{p}}^{2}=0,083$; Fähigkeitsselbstkonzept, $F(1 ; 746)=45.169 ; p<0,001 ; \mathrm{n}_{\mathrm{p}}{ }^{2}=0,057$; Selbstwirksamkeit, $F(1 ; 746)=72.356 ; p<0,001 ; \eta_{\mathrm{p}}^{2}=0,088$; Fachwissen, $F(1 ; 746)=248.333 ; p<0,001 ; \eta_{\mathrm{p}}^{2}=0,250$. Darüber hinaus ergeben sich für die motivationalen Regulationsstile folgende Haupteffekte für Schulform: intrinsische, $F(1 ; 746)=31.485 ; p<0,001 ; \eta_{\mathrm{p}}{ }^{2}=0,040$; identifizierte, $F(1 ; 746)=31.110 ; p<0,001 ; \eta_{\mathrm{p}}{ }^{2}=0,040$; introjizierte, $F(1 ; 746)=39.662 ; p<0,001 ; \eta_{\mathrm{p}}{ }^{2}=0,050$; und extrinsische Motivation, $F(1 ; 746)=24.954 ; p<0,001 ; \eta_{p}{ }^{2}=0,032$. Die hierbei genannten Haupteffekte sind klein bis groß. Aufgrund der Formulierung von $\mathrm{H} 1$ werden an dieser Stelle keine weiteren Haupteffekte und Interaktionen berichtet. Diese sind jedoch im weiteren Verlauf der Ergebnisdarstellung aufgeführt.

Tab. 2 Auflistung der signifikanten Unterschiede mit Effektstärken zwischen gymnasialen und nicht-gymnasialen Schulformen für die erhobenen Konstrukte zu einzelnen MZP

\begin{tabular}{|c|c|c|c|c|c|c|}
\hline & & $\begin{array}{l}\text { Mittelwerte } \\
\text { gymnasial/n }\end{array}$ & $\begin{array}{l}\text { Standardabweichung } \\
\text { ial }\end{array}$ & Teststatistik & $p$ & $\begin{array}{l}\text { Effektstärke } \\
\text { Cohens } d\end{array}$ \\
\hline \multirow{3}{*}{$\begin{array}{l}\text { Einstellung zu } \\
\text { MINT-Berufen }\end{array}$} & $\mathrm{t}_{0}$ & $2,68 / 2,30$ & $0,59 / 0,58$ & 68.420 & $<0,001$ & 0,648 \\
\hline & $\mathrm{t}_{1}$ & $2,70 / 2,39$ & $0,62 / 0,59$ & 42.681 & $<0,001$ & 0,508 \\
\hline & $\mathrm{t}_{2}$ & $2,68 / 2,32$ & $0,65 / 0,57$ & 55.091 & $<0,001$ & 0,576 \\
\hline \multirow{3}{*}{$\begin{array}{l}\text { Fähigkeitsselbst- } \\
\text { konzept }\end{array}$} & $\mathrm{t}_{0}$ & $2,93 / 2,60$ & $0,64 / 0,66$ & 42.977 & $<0,001$ & 0,510 \\
\hline & $\mathrm{t}_{1}$ & $2,95 / 2,64$ & $0,66 / 0,70$ & 33.429 & $<0,001$ & 0,460 \\
\hline & $\mathrm{t}_{2}$ & $2,96 / 2,66$ & $0,66 / 0,71$ & 33.533 & $<0,001$ & 0,443 \\
\hline \multirow[t]{3}{*}{ Selbstwirksamkeit } & $\mathrm{t}_{0}$ & $2,37 / 2,20$ & $0,55 / 0,58$ & 14.960 & $<0,001$ & 0,304 \\
\hline & $\mathrm{t}_{1}$ & $3,16 / 2,81$ & $0,45 / 0,58$ & 79.701 & $<0,001$ & 0,706 \\
\hline & $\mathrm{t}_{2}$ & $2,99 / 2,57$ & $0,53 / 0,69$ & 84.784 & $<0,001$ & 0,716 \\
\hline \multirow{3}{*}{$\begin{array}{l}\text { Intrinsische } \\
\text { Motivation }\end{array}$} & $\mathrm{t}_{0}$ & $2,78 / 2,46$ & $0,67 / 0,72$ & 34.500 & $<0,001$ & 0,466 \\
\hline & $\mathrm{t}_{1}$ & $2,79 / 2,54$ & $0,72 / 0,79$ & 17.689 & $<0,001$ & 0,336 \\
\hline & $\mathrm{t}_{2}$ & $2,69 / 2,39$ & $0,78 / 0,77$ & 23.802 & $<0,001$ & 0,386 \\
\hline \multirow{3}{*}{$\begin{array}{l}\text { Identifizierte } \\
\text { Motivation }\end{array}$} & $\mathrm{t}_{0}$ & $2,49 / 2,22$ & $0,73 / 0,67$ & 24.906 & $<0,001$ & 0,380 \\
\hline & $\mathrm{t}_{1}$ & $2,53 / 2,33$ & $0,68 / 0,69$ & 13.816 & $<0,001$ & 0,293 \\
\hline & $\mathrm{t}_{2}$ & $2,45 / 2,16$ & $0,71 / 0,74$ & 26.712 & $<0,001$ & 0,404 \\
\hline \multirow{3}{*}{$\begin{array}{l}\text { Introjizierte } \\
\text { Motivation }\end{array}$} & $\mathrm{t}_{0}$ & $2,07 / 2,37$ & $0,71 / 0,74$ & 29.857 & $<0,001$ & 0,417 \\
\hline & $\mathrm{t}_{1}$ & $2,06 / 2,41$ & $0,74 / 0,75$ & 36.116 & $<0,001$ & 0,471 \\
\hline & $\mathrm{t}_{2}$ & $2,07 / 2,34$ & $0,76 / 0,77$ & 20.950 & $<0,001$ & 0,354 \\
\hline \multirow{3}{*}{$\begin{array}{l}\text { Extrinsische } \\
\text { Motivation }\end{array}$} & $\mathrm{t}_{0}$ & $2,14 / 2,39$ & $0,72 / 0,73$ & 20.157 & $<0,001$ & 0,346 \\
\hline & $\mathrm{t}_{1}$ & $2,12 / 2,43$ & $0,72 / 0,73$ & 29.978 & $<0,001$ & 0,429 \\
\hline & $\mathrm{t}_{2}$ & $2,21 / 2,38$ & $0,71 / 0,71$ & 9952 & 0,002 & 0,239 \\
\hline \multirow[t]{2}{*}{ Fachwissen } & $\mathrm{t}_{1}$ & $0,80 / 0,61$ & $0,15 / 0,22$ & 172.870 & $<0,001$ & 1,080 \\
\hline & $\mathrm{t}_{2}$ & $0,75 / 0,56$ & $0,17 / 0,22$ & 171.048 & $<0,001$ & 1,012 \\
\hline
\end{tabular}


Zur näheren Betrachtung der Unterschiede zwischen den Schulformen für jeden einzelnen MZP wird eine einfaktorielle ANOVA mit dem Faktor Schulform durchgeführt und die jeweiligen Effektstärken ermittelt (Tab. 2).

\section{Entwicklung der Einstellung zu MINT-Berufen über die MZP (H2)}

Die dreifaktorielle ANOVA liefert neben dem Haupteffekt für Schulform die beiden Haupteffekte für MZP, $F(2 ; 1492)=5377 ; p=0,005 ; \eta_{\mathrm{p}}^{2}=0,007$; und für Untersuchungsgruppen, $F(2 ; 746)=3195 ; p=0,042 ; \eta_{p}^{2}=0,008$; jeweils mit einem sehr kleinen Effekt, jedoch keine Interaktionen zwischen den Faktoren, übergreifend $F \leq 2025$; $p \geq 0,132$. Somit gibt es zwischen den Untersuchungsgruppen und zwischen den beiden Schulformen keine signifikanten Unterschiede der Entwicklung der Einstellung zu MINT-Berufen über die MZP.

\section{Nachhaltigkeit des Fachwissens (H3)}

Die dreifaktorielle ANOVA zeigt neben dem Haupteffekt für Schulform sowohl die beiden Haupteffekte für MZP, $F(1 ; 746)=38.542 ; p<0,001 ; \eta_{p}{ }^{2}=0,049$; und für Untersuchungsgruppen, $F(2 ; 746)=22.071 ; p<0,001$; $\mathrm{n}_{\mathrm{p}}{ }^{2}=0,056$; als auch die Interaktionen zwischen MZP und Untersuchungsgruppen, $F(2 ; 746)=3436 ; p=0,033$; $\eta_{\mathrm{p}}{ }^{2}=0,009 ; \quad$ zwischen Schulform und Untersuchungsgruppen, $F(2 ; 746)=7664 ; p=0,001 ; \eta_{p}^{2}=0,020$; sowie zwischen MZP, Schulform und Untersuchungsgruppen, $F(2 ; 746)=3760 ; p=0,024 ; \eta_{\mathrm{p}}{ }^{2}=0,010$; jedoch keine Interaktion zwischen MZP und Schulform, $F<1$.

Wird nun die Entwicklung des Fachwissens zwischen dem Post- und Follow-up-Test für die beiden Schulformen separat für die einzelnen Untersuchungsgruppen betrachtet (Abb. 1), so lässt sich feststellen, dass bei der gymnasialen Substichprobe bei KG das Fachwissen signifikant abfällt, $F(1 ; 170)=54.095 ; p<0,001 ; \eta_{\mathrm{p}}{ }^{2}=0,241$; bei den beiden Experimentalgruppen jedoch stabil bleibt. Im Falle der nicht-gymnasialen Substichprobe findet sowohl bei KG, $F(1 ; 76)=4465 ; p=0,038 ; \eta_{\mathrm{p}}^{2}=0,055 ;$ als auch bei EG1, $F(1 ; 105)=17.496 ; p<0,001 ; \eta_{\mathrm{p}}{ }^{2}=0,143$; eine signifikante Abnahme des Fachwissens statt, welches lediglich bei EG2 stabil bleibt.

Anhand einer einfaktoriellen ANOVA wird zudem erkennbar, dass im Falle der gymnasialen Schulform alle Untersuchungsgruppen im Posttest keine signifikanten Unterschiede zwischen den Ergebnissen des Wissenstests zeigen und sich die signifikanten Unterschiede zwischen $\mathrm{KG}$ und den Experimentalgruppen erst im Follow-up-Test herausstellen (Abb. 1). Bei der nicht-gymnasialen Substichprobe ist bereits im Posttest das Wissen der KG signifikant nied-

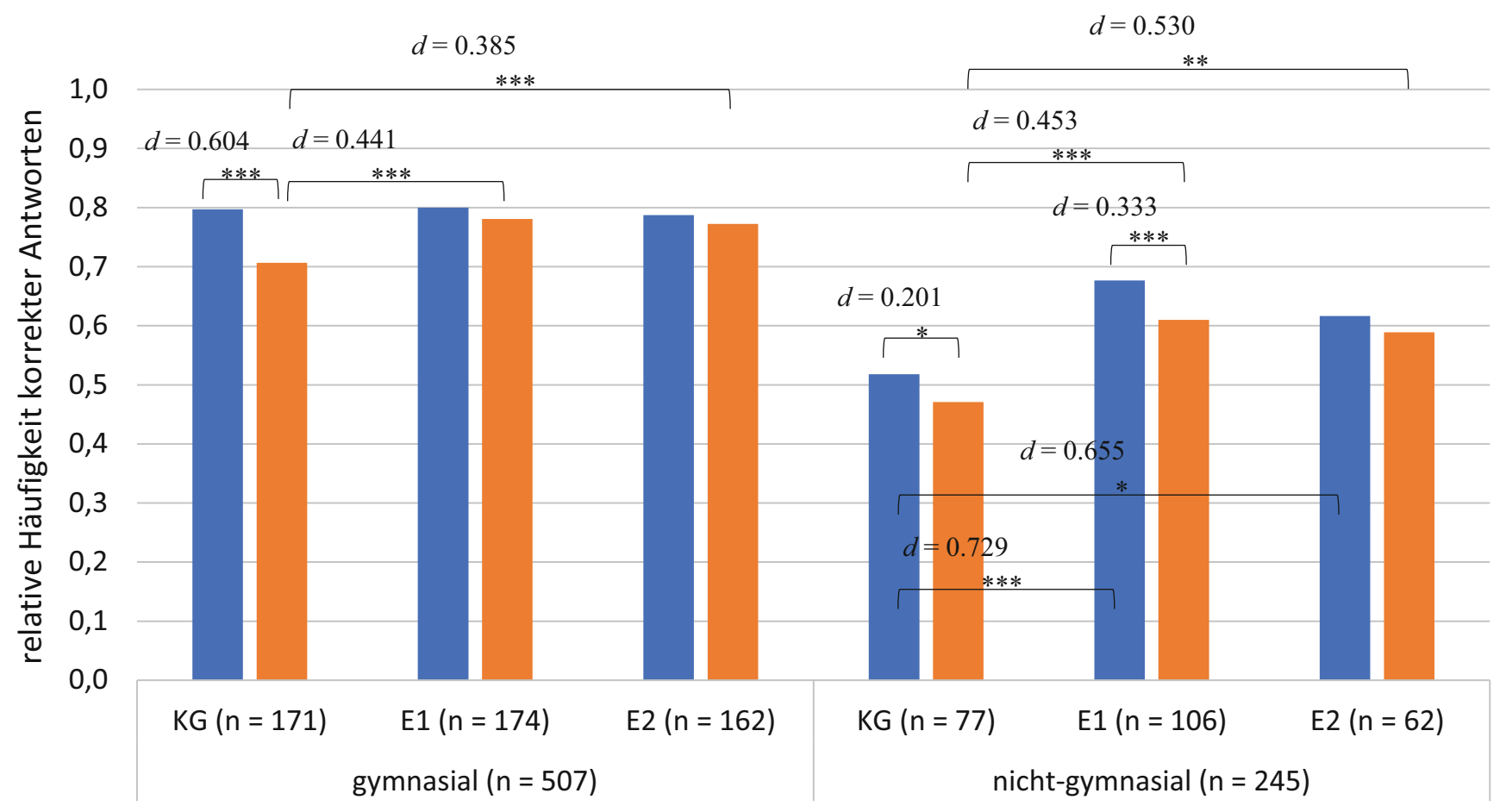

$\square \mathrm{t} 1 \quad \mathrm{t} 2$

Abb. 1 Darstellung der Ausprägungen des Konstrukts Fachwissen für die beiden Schulformen und die einzelnen Untersuchungsgruppen (Signifikanzlevel: * $p<0,05 ; * * p<0,01 ; * * * p<0,001)$ 
riger ausgeprägt als bei den beiden Experimentalgruppen, was sich ebenfalls im Follow-up-Test zeigt (Abb. 1).

\section{Entwicklung des Fähigkeitsselbstkonzepts über die MZP (H4a)}

Aus der dreifaktoriellen ANOVA lässt sich erkennen, dass es neben dem bereits bekannten Haupteffekt für Schulform noch einen Haupteffekt für Untersuchungsgruppen, $F(2 ; 746)=4681 ; p=0,010 ; \eta_{p}{ }^{2}=0,012 ;$ mit einer sehr kleinen Effektstärke, jedoch keinen Haupteffekt für MZP, $F(2 ; 1492)=2380 ; p=0,093$; sowie keine Interaktionen zwischen den Faktoren, übergreifend $F \leq 2295 ; p \geq 0,057$; gibt. Somit gibt es keine Entwicklung des Konstrukts über die MZP.

\section{Entwicklung der intrinsischen Motivation über die MZP (H4b)}

Die dreifaktorielle ANOVA zeigt neben dem Haupteffekt für Schulform die beiden Haupteffekte für MZP, $F(2 ; 1492)=18.438 ; p<0,001 ; \eta_{p}{ }^{2}=0,024$; und für Untersuchungsgruppen, $F(2 ; 746)=7748 ; p<0,001 ; \eta_{\mathrm{p}}{ }^{2}=0,020$; jedoch keine Interaktionen zwischen den Faktoren, übergreifend $F \leq 2964 ; p \geq 0,052$. Aufgrund der fehlenden Interaktionen zwischen dem MZP und den anderen beiden Faktoren ist aus methodischer Sicht eine nähere Betrachtung der zeitlichen Entwicklung der intrinsischen Motivation lediglich schulform- und untersuchungsgruppenübergrei- fend sinnvoll. Hierbei lässt sich eine signifikant niedrigere Ausprägung des Konstrukts im Prätest im Vergleich zum Posttest, $p=0,032 ; d=0,048$; sowie im Follow-up-Test im Vergleich zum Prätest, $p=0,001 ; d=0,113$; und Posttest, $p<0,001 ; d=0,154$; erkennen. Aufgrund der sehr kleinen Effektstärke kann hierbei aber nahezu von gleichbleibender Ausprägung der intrinsischen Motivation über die MZP gesprochen werden.

\section{Entwicklung der Selbstwirksamkeit über die MZP (H5)}

Die dreifaktorielle ANOVA liefert neben dem Haupteffekt für Schulform die beiden Haupteffekte für MZP, $F(2 ; 1492)=589.032 ; p<0,001 ; \eta_{p}{ }^{2}=0,441$; und für Untersuchungsgruppen, $F(2 ; 746)=9026 ; p<0,001 ; n_{p}{ }^{2}=0,024$; sowie die Interaktionen zwischen MZP und Schulform, $F(2 ; 1492)=19.745 ; p<0,001 ; \eta_{\mathrm{p}}{ }^{2}=0,026 ;$ zwischen MZP und Untersuchungsgruppen, $F(4 ; 1492)=3658 ; p=0,006$; $\eta_{p}{ }^{2}=0,010 ;$ und zwischen Untersuchungsgruppen und Schulform, $F(2 ; 746)=5017 ; p=0,007 ; \eta_{\mathrm{p}}{ }^{2}=0,013$; jedoch keine Dreifachinteraktion, $F(4 ; 1492)=1876 ; p=0,112$. Zur näheren Betrachtung der Entwicklung der Selbstwirksamkeit über die MZP wird für die einzelnen Untersuchungsgruppen und die beiden Schulformen jeweils eine einfaktorielle ANOVA mit dem Faktor MZP durchgeführt. Die Berechnungen zeigen für beide Schulformen und alle drei Untersuchungsgruppen dasselbe Ergebnis (Abb. 2), wobei eine starke signifikante Zunahme vom Prä- zum

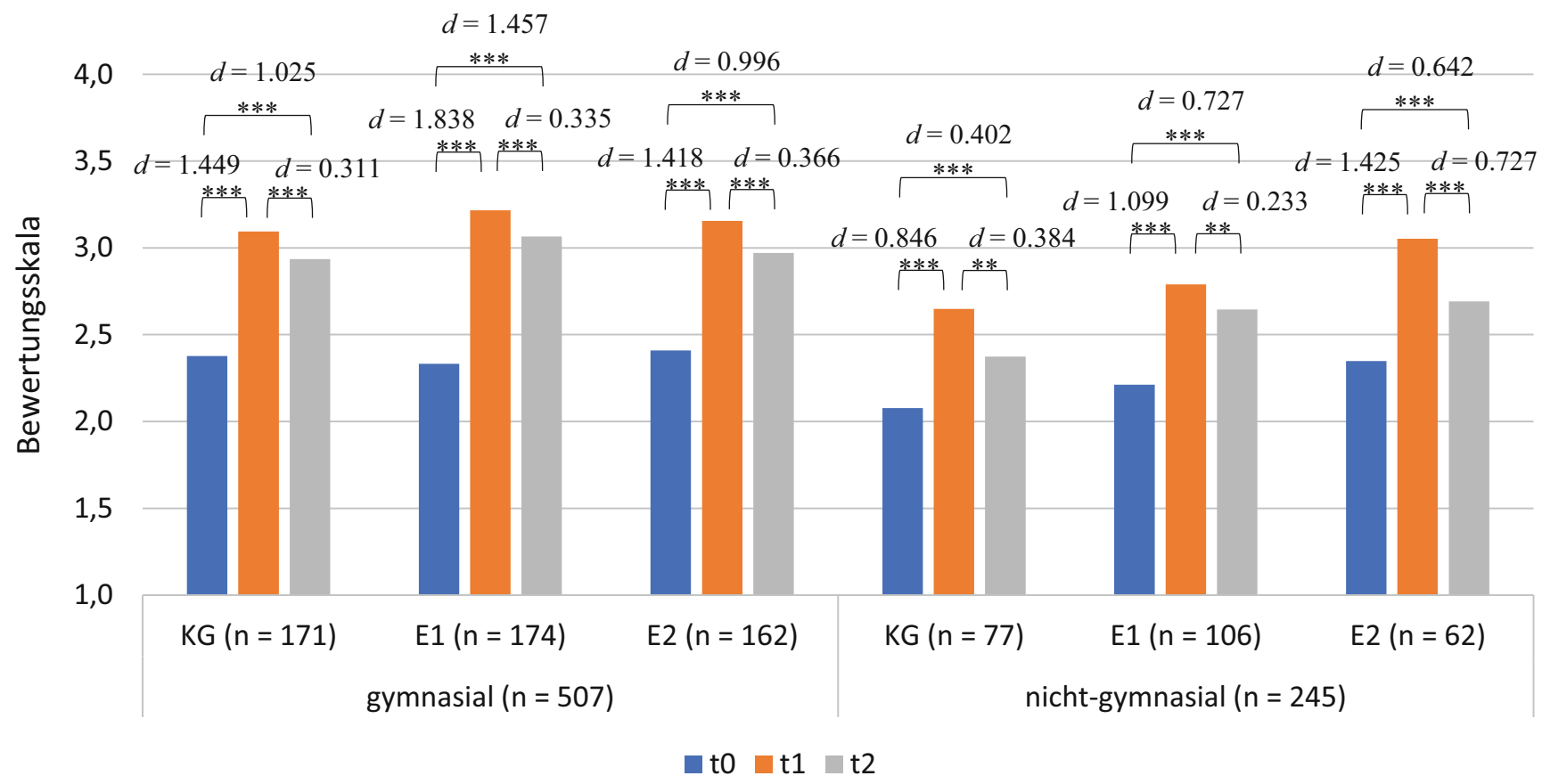

Abb. 2 Darstellung der Ausprägungen des Konstrukts Selbstwirksamkeit für die beiden Schulformen und die einzelnen Untersuchungsgruppen (Signifikanzlevel: * $p<0,05 ; * * p<0,01 ; * * * p<0,001$ ) 
Posttest und anschließend eine geringe signifikante Abnahme vom Post- zum Follow-up-Test erfolgen. Dennoch ist die Ausprägung der Selbstwirksamkeit im Follow-up-Test signifikant höher als im Prätest.

\section{Diskussion}

Die Studie hatte das Ziel, die Wirksamkeit einer schulischen Vor- und Nachbereitung mit und ohne integrierte Aspekte der Berufsorientierung zu einem Schülerlaborprogramm zur Nanotechnologie im Hinblick auf Einstellung zu MINT-Berufen, Fähigkeitsselbstkonzept, motivationale Regulation, Selbstwirksamkeit und Fachwissen zu untersuchen. Die Ergebnisse entsprechen nur teilweise den Hypothesen und zeigen unterschiedliche Auswirkungen des konzipierten Schülerlaborprogramms und der schulischen Vorund Nachbereitung auf die einzelnen Konstrukte.

\section{Vergleich der Konstrukte zwischen den Schulformen zu allen MZP (H1)}

Die Ergebnisse zeigen für alle Konstrukte signifikante Unterschiede zwischen der gymnasialen und nicht-gymnasialen Schulform (Tab. 2) mit schwachen bis mittleren Effekten zwischen 0,239 und 0,716, wobei einzig die Konstrukte introjizierte und extrinsische Motivation bei der nicht-gymnasialen Schulform und alle anderen Konstrukte bei der gymnasialen Schulform signifikant höhere Werte aufweisen. Der Vergleich des Konstrukts Fachwissen zwischen den Schulformen weist zu beiden einbezogenen MZP sehr hohe Effektstärken $(>1,0)$ auf, was möglicherweise unter anderem auf das traditionell unterschiedlich intendierte fachinhaltliche Leistungsniveau in den beiden Schulformen zurückzuführen sein könnte. So scheinen die unterschiedlichen Schulformen einen starken Einfluss auf die Lernleistung und -motivation zu haben.

Die Ergebnisse und die Bestätigung der H1 erweisen sich als literaturkonform (Wirth 2019). Zudem scheint die unterschiedliche Unterrichtsgestaltung die motivationale Ausrichtung zu bedingen, wobei nicht-gymnasiale Lernende schwächer ausgeprägte selbstbestimmte Regulationsstile aufweisen als die gymnasialen Schülerinnen und Schüler. Da sich die extrinsischen und intrinsischen Regulationsstile nicht ausschließen, sondern kumulativ die Ausprägung der Lernmotivation darstellen (Müller et al. 2007), kann an dieser Stelle nicht darauf geschlossen werden, dass gymnasiale Lernende intrinsisch und identifiziert und nichtgymnasiale Lernende extrinsisch und introjiziert geleitet werden. Jedoch geben die Ergebnisse einen Hinweis auf die unterschiedliche Stärke der Ausprägung der motivationalen Regulationsstile in Abhängigkeit zur Darbietungsart der Lerngelegenheiten in der Schule.

\section{Entwicklung der Einstellung zu MINT-Berufen über die MZP (H2)}

Des Weiteren haben den Ergebnissen zufolge die berufsorientierenden Aspekte in der schulischen Vor- und Nachbereitung keine Auswirkungen auf die berufliche Ausrichtung und Entwicklung der Teilnehmenden. Die Lernenden scheinen unabhängig von der Untersuchungsgruppe mit anfangs stark positiver bzw. negativer Einstellung zu MINT-Berufen durch die Intervention in ihrer beruflichen Ausrichtung bestätigt zu werden, wodurch keine Änderung der Einstellung erfolgt. Somit wird H2 entgegen dem Stand der Forschung (Sasson 2019; Weßnigk 2013) lediglich für EG1 und KG bestätigt.

Ein Grund kann der geringe zeitliche Umfang der expliziten Berufsorientierung in diesem Programm sein, welche in vielen anderen Studien (Weßnigk 2013; Haucke 2013) in Art und Umfang deutlich stärker ausgeprägt und damit zeitlich intensiver sowie anschaulicher gestaltet wurde als die explizite Präsentation der Inhalte in dieser Studie. Ferner ist nicht auszuschließen, dass die herangezogenen naturwissenschaftlichen Ausbildungsberufe die Lernenden nicht ausreichend angesprochen und diese sich somit lediglich oberflächlich mit deren Beschreibungen sowie Einsatz- und Anwendungsbereichen beschäftigt haben. Es scheint deutlich zu werden, dass eine möglicherweise zu oberflächliche Beschäftigung mit naturwissenschaftlichen Berufen nicht ausreicht. Vielmehr könnte in der schulischen Vor- und Nachbereitung eine Beschäftigung mit verschiedenen Berufen durch authentische Einblicke in Berufsfelder bzw. in einen Berufsalltag dazu führen, dass sich Jugendliche überhaupt erst ein umfassendes Bild eines Berufs machen können. Diese Immersion wird auch bei weniger gut qualifizierten Jugendlichen oder bei Lernenden mit sprachlichen oder fachlichen Hürden empfohlen und über Praktika z.T. schon umgesetzt (vgl. acatech und Körber-Stiftung 2020). Damit zusammen hängt auch die Art der Darbietung der berufsorientierenden Aspekte, da eine derartige Authentizität in der Schule ohne mediale Unterstützung nicht geleistet werden kann. So könnten in der Vor- und Nachbereitung z. B. Kurzvideos in Videoportalen mit personenbezogenen Vorbildern eingesetzt werden. Haucke (2013) stellt z.B. die Rolle des Fernsehens für die Berufswahl heraus. Durch die sich wandelnde Art der Mediennutzung und Informationsbeschaffung durch die Schülerinnen und Schüler sollte heute das Internet nicht vernachlässigt werden. Eine repräsentative Umfrage unter 12- bis 19-Jährigen hat ergeben, dass YouTube-Videos bei den Befragten ein hohes Aktivierungspotenzial hinsichtlich praktischer, kultureller Tätigkeiten besitzt, sofern diese Webvideos als unterhaltsam, witzig und neu/zeitgemäß empfunden werden (Rat für kulturelle Bildung 2019). Sobald die Internetmedien aber über die Aktivierung hinausgehend genutzt werden sollen, stellt 
sich die Frage, inwieweit Jugendliche die präsentierten Inhalte auch einschätzen können - das MINT Nachwuchsbarometer 2020 sagt z. B. aus, dass nur $14 \%$ der Abiturientinnen und Abiturienten ,systematisch nach Informationen im Netz suchen und diese hinsichtlich ihrer Glaubwürdigkeit beurteilen [können]“ (acatech und Körber-Stiftung 2020, S. 11). Da sich diese Prozentzahl vermutlich auch in der Gruppe der Jugendlichen mit anderem Schulabschluss widerspiegeln wird, benötigen Jugendliche auch bei der Suche und Auswertung von Informationen zur Berufsorientierung im Internet Unterstützung in der analogen Welt.

Auch wenn in der Vor- und Nachbereitung nur MINTBerufe mit Bezug zur Nanotechnologie vorgestellt worden sind, so lässt sich vermuten, dass Schülerinnen und Schüler Schwierigkeiten dabei haben, das im Schülerlabor präsentierte Wissen auf hypothetisch beschriebene Situationen verschiedener Berufsfelder zu übertragen. Dieser fehlende Transfer ist z. B. auch von Gropengießer (1995) gezeigt worden, da Lernende fachliche biologische Inhalte nicht mit ihrem später intendierten Beruf zusammenbringen konnten oder vermuteten, das die fachlichen Lerninhalte im Beruf nicht angewendet würden. Auch dieser Aspekt wäre sicher durch eine authentischere Präsentation der benötigten Inhalte besser adressierbar.

Gemäß der erhobenen Daten streben knapp $80 \%$ der Probanden - sowohl gymnasiale als auch nicht-gymnasiale Lernende - das Abitur als Schulabschluss an, was ein stärkeres Interesse an akademischen Berufen vermuten lässt, wodurch die bei EG2 eingeführten nicht-akademischen Ausbildungsberufe keine positive Wirkung auf die Schülerinnen und Schüler haben könnten. Zudem sind die Lernenden der Gemeinschaftsschule im Mittel noch über ein Jahr, die des Gymnasiums im Mittel noch über 3 Jahre von dem Zeitpunkt ihrer eigentlichen Berufswahl entfernt, wodurch berufsorientierenden Aspekten ggf. nicht so viel Aufmerksamkeit zukommt wie fachlichen Inhalten. Dies ist insbesondere im Vergleich zu den Ergebnissen des Fachwissenstests bei der gymnasialen Substichprobe ersichtlich, bei der die Lernenden noch drei Monate nach der Intervention den Wissenstest zu 70-80\% korrekt lösen konnten. Ebenso könnte es sein, dass der Berufswahlprozess zu dem Zeitpunkt ggf. noch nicht ernsthaft begonnen hat - durch eine dann nicht erfolgende subjektive Auseinandersetzung mit den Berufen ist vermutlich auch keine Änderung der Einstellungen $\mathrm{zu}$ erwarten.

\section{Nachhaltigkeit des Fachwissens (H3)}

Gemäß den Ergebnissen kann H3 für die gymnasiale Substichprobe bestätigt, für die nicht-gymnasiale Substichprobe jedoch ausschließlich für KG und EG2 nachgewiesen werden. Dabei wird durch die einfaktorielle ANOVA erkennbar, dass im Falle der gymnasialen Schulform alle Untersu- chungsgruppen gleichermaßen das im Schülerlabor dargebotene Wissen aufgenommen haben, da im Posttest keine signifikanten Unterschiede zwischen den Ergebnissen des Wissenstests vorliegen. Dass sich die signifikanten Unterschiede zwischen der KG und den Experimentalgruppen erst im Follow-up-Test herausstellen, weist darauf hin, dass eine schulische Vor- und Nachbereitung im Allgemeinen der Nachhaltigkeit der Wissenssicherung dient. Auch andere Arbeiten (Wilde und Bätz 2006; Guderian 2007; Klees und Tillmann 2015) weisen darauf hin, dass die Effektivität und Nachhaltigkeit der gelernten Inhalte durch eine Vorbereitung eines Besuchs am außerschulischen Lernort erhöht werden. Zudem spielt die detaillierte Wiederholung der gelernten Inhalte im Rahmen der schulischen Nachbereitung zur Förderung und Sicherung des Fachwissens der beiden Experimentalgruppen eine wichtige Rolle.

Bezüglich der Nachhaltigkeit des Fachwissens bei der nicht-gymnasialen Schulform hingegen scheint die schulische Vor- und Nachbereitung mit integrierten Aspekten der Berufsorientierung wirksamer zu sein, wobei der Wissenstest bei EG1 und KG im Follow-up-Test signifikant niedrigere Ergebnisse zeigt als im Posttest. Ein Grund hierzu könnte sein, dass gerade durch die Verknüpfung der fachlichen Inhalte mit berufsorientierenden Aspekten den Lernenden die Tragfähigkeit der Nanotechnologie bewusst wird und sie hierdurch das erlangte Wissen nachhaltiger speichern können.

Aufgrund der vermehrten Teilnahme von Gymnasien an außerschulischen Veranstaltungen scheinen die Novelty Space Effekte weniger Auswirkungen auf deren Lernende zu haben. Bei der nicht-gymnasialen Substichprobe hingegen könnte der im Posttest signifikant niedrigere Wissensstand auf die vermeintlich hohen Novelty Space Effekte zurückzuführen sein, wodurch sich die Lernenden der nicht vertrauten Umgebung wegen vorerst weniger auf die dargebotenen Lerninhalte konzentrieren können (Orion und Hofstein 1994).

\section{Entwicklung des Fähigkeitsselbstkonzepts über die MZP (H4a)}

Die Ergebnisse zeigen für die beiden Schulformen keine Entwicklung des Fähigkeitsselbstkonzepts über die MZP, wodurch H4 im Hinblick auf dieses Konstrukt nicht bestätigt werden kann. Dieses Ergebnis ist nicht konform mit dem Stand der Forschung, da diverse Studien nachweisen konnten, dass bereits ein alleiniger Besuch an außerschulischen Lernorten zu einer kurz- bis langfristigen positiven Entwicklung des Fähigkeitsselbstkonzepts führt (Wirth 2019; Weßnigk 2013; Pawek 2009).

Da dieses Konstrukt einen eindeutigen Zusammenhang mit der Berufsorientierung der Lernenden aufweist (Baumert 1992; Filipp 2006), ist dieses Ergebnis jedoch im Rah- 
men dieser Studie mit den Ausführungen zum Konstrukt Einstellung zu MINT-Berufen vergleichbar.

\section{Entwicklung der intrinsischen Motivation über die MZP (H4b)}

Die Ergebnisse zeigen bei der intrinsischen Motivation jeweils untersuchungsgruppen- und schulformübergreifend eine positive Entwicklung zwischen dem Prä- und Posttest, wobei sich dieses Konstrukt ebenfalls durch eine fachliche sowie berufsorientierte schulische Vor- und Nachbereitung nicht langfristig stabilisieren lässt. Dies ist vor allem an der nicht vorhandenen Interaktion zwischen Untersuchungsgruppen und anderen herangezogenen Faktoren ersichtlich.

Somit lässt sich H4 im Hinblick auf das Konstrukt intrinsische Motivation lediglich beim Prä- und Posttest bestätigen. Dieses Ergebnis entspricht nur teils dem theoretischen Hintergrund, da hiernach die intrinsische Motivation durch einen Schülerlaborbesuch gesteigert (Braund und Reiss 2006; Nickolaus et al. 2018) und durch eine zusätzliche Verknüpfung schulischer und außerschulischer Lernorte nachhaltig gesichert werden sollte (Weßnigk 2013; Guderian und Priemer 2008). Jedoch zeigen sich auch an dieser Stelle gemäß dem literaturkonformen Zusammenhang zwischen der intrinsischen Motivation und der Berufswahl der Lernenden (Taskinen et al. 2013) vergleichbare Auswertungen mit dem hierbei erhobenen Konstrukt Einstellung zu MINT-Berufen.

\section{Entwicklung der Selbstwirksamkeit über die MZP (H5)}

Bei dem Konstrukt Selbstwirksamkeit lässt sich eine klare Entwicklung über die MZP erkennen (Abb. 2). Gemäß den berichteten Interaktionen unterscheiden sich die Ausprägungen über die MZP zwischen den Untersuchungsgruppen und den Schulformen, jedoch weist die zeitliche Entwicklung bei allen Vergleichsgruppen eine vergleichbare Tendenz auf. Somit lässt sich H5 bestätigen.

Es ist anzumerken, dass bei allen Untersuchungsgruppen der gymnasialen Schulform die signifikante Zunahme der Selbstwirksamkeit vom Prä- zum Posttest sowie vom Präzum Follow-up-Test mit sehr hohen Effektstärken zwischen 0,996 und 1,838 einhergehen, wohingegen die signifikanten Abnahmen vom Post- zum Follow-up-Test lediglich geringe Effektstärken zwischen 0,311 und 0,366 aufweisen (Abb. 2). Hieraus lässt sich schlussfolgern, dass die Lernenden unmittelbar nach der Intervention einen eindeutigen Zuwachs der Selbstwirksamkeit wahrnehmen und das Gefühl haben, die neu gewonnenen Erkenntnisse langfristig behalten zu haben. Diese Wahrnehmung wird vor allem bei den beiden Experimentalgruppen durch die dargelegten Ergebnisse des Fachwissenstests unterstützt, bei dem kei- ne signifikanten Unterschiede vom Post- zum Follow-upTest festgestellt werden konnten. Bei der nicht-gymnasialen Substichprobe können derartige Erkenntnisse und Verknüpfungen zum Konstrukt Fachwissen nicht gezogen werden, da hierbei zwar ebenfalls eine starke Zunahme der Selbstwirksamkeit vom Prä- zum Posttest vorliegt, jedoch aber insbesondere bei EG2 eine starke Abnahme folgt.

\section{Zusammenfassung}

Zusammenfassend lässt sich festhalten, dass die beiden ersten Forschungsfragen mit der Analyse der erhobenen Daten nicht komplett bejaht werden können. Auch hieran ist zu erkennen, dass die berufsorientierenden Aspekte in der schulischen Vor- und Nachbereitung in der dargebotenen Form keinen Mehrwert erbracht haben. Es lässt sich festhalten, dass beide Fassungen nachhaltige Effekte hinsichtlich der Konstrukte Fachwissen und Selbstwirksamkeit zeigen, jedoch in dieser Form keinen Einfluss auf die Einstellung zu MINT-Berufen, die intrinsische Motivation und das Fähigkeitsselbstkonzept haben. Hingegen lässt sich FF3 vollständig bejahen.

Somit konnte die Wirksamkeit der hier durchgeführten Verknüpfung zwischen schulischen und außerschulischen Lernorten lediglich im Hinblick auf die kognitiven Konstrukte der erhobenen Daten nachgewiesen werden, wobei die Integration der berufsorientierenden Aspekte kaum Mehrwert bringt.

\section{Limitation und Ausblick}

Im Rahmen der durchgeführten Studie sind aufgrund der äußeren Gegebenheiten einige Limitationen zu verzeichnen. So konnte aufgrund der zeitversetzten Anmeldung zu den Schülerlabortagen keine randomisierte Stichprobe geschaffen werden, wobei lediglich eine händische und schrittweise Zuordnung zu den einzelnen Untersuchungsgruppen möglich war.

Zum durchgeführten Fachwissenstest ist anzumerken, dass dieser für das Gymnasium tendenziell zu leicht ausgefallen ist und Deckeneffekte zu beobachten sind. Die ursprüngliche Zielgruppe des Schülerlaborprogramms war die Gemeinschaftsschule. Da Gemeinschaftsschulen zu Beginn der Erhebung das Angebot weniger stark nutzten als erhofft, wurde eine Öffnung auch für gymnasiale Klassen entschieden. Wegen der Vergleichbarkeit wurden dann aber die in Zusammenarbeit mit Gemeinschaftsschullehrkräften für die ursprüngliche Zielgruppe konzipierten und schon in einigen Klassen eingesetzten Instrumente beibehalten, was zu den beobachteten sehr hohen Mittelwerten bei den Gymnasialklassen geführt hat. 
Des Weiteren wäre eine interaktivere, authentischere und vielseitiger gestaltete sowie zeitlich umfassendere und detailliertere berufsorientierte schulische Vor- und Nachbereitung voraussichtlich zielführender und untersuchungswert.

Gemäß der erlangten Ergebnisse könnte es sich lohnen, die Karrierechancen und das Image von Ausbildungsberufen stärker hervorzuheben bzw. das Angebot auch auf akademische Berufe zu erweitern. Ebenfalls wäre ggf. eine ausschließlich berufsorientierende schulische Vor- und Nachbereitung im Rahmen der Entwicklung der Einstellung zu MINT-Berufen sinnvoller und zielführender, da sich hierbei die Lernenden ausgiebiger auf die Berufsorientierung konzentrieren, detailliertere Sachverhalte erarbeiten und interaktivere Herangehensweisen nutzen könnten sowie mehr zeitliche Ressourcen hätten, die für sich interessanten und vorteilhaften Aspekte herauszufinden.

Ebenfalls wäre der Effekt einer freien und weniger geleiteten Erarbeitung der fachlichen Inhalte an den einzelnen Experimentierstationen fachdidaktisch interessant, da hierbei gemäß dem Stand der Forschung eine stärkere Entwicklung der intrinsischen Motivation folgen sollte.

Förderung Die Durchführung des Vorhabens wurde mit Mitteln in der Förderlinie strategische Vernetzung des Leibniz-Wettbewerbs im Rahmen des Leibniz-WissenschaftsCampus „Kiel Science Outreach Campus" (KiSOC) ermöglicht. Die Autoren danken zudem der Kieler Forschungswerkstatt und der Ludwig-Maximilians-Universität München, in dessen Rahmen das Schülerlaborprogramm schülergerecht gestaltet und aktiv umgesetzt werden konnte.

Funding Open Access funding enabled and organized by Projekt DEAL.

Open Access Dieser Artikel wird unter der Creative Commons Namensnennung 4.0 International Lizenz veröffentlicht, welche die Nutzung, Vervielfältigung, Bearbeitung, Verbreitung und Wiedergabe in jeglichem Medium und Format erlaubt, sofern Sie den/die ursprünglichen Autor(en) und die Quelle ordnungsgemäß nennen, einen Link zur Creative Commons Lizenz beifügen und angeben, ob Änderungen vorgenommen wurden.

Die in diesem Artikel enthaltenen Bilder und sonstiges Drittmaterial unterliegen ebenfalls der genannten Creative Commons Lizenz, sofern sich aus der Abbildungslegende nichts anderes ergibt. Sofern das betreffende Material nicht unter der genannten Creative Commons Lizenz steht und die betreffende Handlung nicht nach gesetzlichen Vorschriften erlaubt ist, ist für die oben aufgeführten Weiterverwendungen des Materials die Einwilligung des jeweiligen Rechteinhabers einzuholen.

Weitere Details zur Lizenz entnehmen Sie bitte der Lizenzinformation auf http://creativecommons.org/licenses/by/4.0/deed.de.

\section{Literatur}

acatech, \& Körber-Stiftung (2020). MINT-Nachwuchsbarometer 2020. https://www.koerber-stiftung.de/fileadmin/user_upload/ koerber-stiftung/redaktion/mint_nachwuchsbarometer/pdf/2020/ MINT-Nachwuchsbarometer-2020.pdf. Zugegriffen: 27. Aug. 2020.
Affeldt, F., Weitz, K., Siol, A., Markic, S., \& Eilks, I. (2015). A nonformal student laboratory as a place for innovation in education for sustainability for all students. Education Sciences, 5(3), $238-254$

Bandura, A. (1997). Self-efficacy: the exercise of control. New York: Freeman Press.

Baumert, J. (1992). Koedukation oder Geschlechtertrennung. Zeitschrift für Pädagogik, 38(1), 83-110.

Brandt, A. (2005). Förderung von Motivation und Interesse durch außerschulische Experimentierlabore. Göttingen: Cuvillier.

Braund, M., \& Reiss, M. (2006). Towards a more authentic science curriculum. The contribution of out-of-school learning. International Journal of Science Education, 28(12), 1373-1388.

Burmeister, M., Rauch, F., \& Eilks, I. (2012). Education for Sustainable Development (ESD) and chemistry education. Chemistry Education Research and Practice, 13(2), 59-68.

Dickhäuser, O. (2006). Fähigkeitsselbstkonzepte. Zeitschrift für Pädagogische Psychologie, 20(1/2), 5-8.

Elster, D. (2009). Naturwissenschaftlicher Unterricht und Beruf. Die Einstellungen Jugendlicher. $M N U, 62(1), 4-10$.

Euler, M. (2005). Schülerinnen und Schüler als Forscher: Informelles Lernen im Schülerlabor. Naturwissenschaften im Unterricht. Physik, 16(90), 4-12.

Euler, M., \& Weßnigk, S. (2011). Schülerlabore und die Förderung kreativer Potenziale. Lernen durch Forschen und Entwickeln. Plus Lucis, 19(1-2), 32-38.

Falk, J.H. (1983). Field trips: a look at environmental effect of learning. Journal of Biological Education, 17(2), 137-142.

Falk, J., Martin, W., \& Balling, J. (1978). The novel field trip phenomenon: adjustment to novel settings interferes with task learning. Journal of Research in Science Teaching, 15(2), 127-134.

Filipp, S.-H. (2006). Kommentar zum Schwerpunktthema. Entwicklung von Fähigkeitsselbstkonzepten. Zeitschrift für Pädagogische Psychologie, 20(1/2), 65-72.

Grolnick, W.S., Ryan, R. M., \& Deci, E. L. (1991). Inner resources for school achievement: Motivational mediators of children's perceptions of their parents. Journal of Educational Psychology, 83(4), $508-517$.

Gropengießer, I. (1995). Biologie für den beruflichen Alltag. Naturwissenschaften im Unterricht Biologie, 19(210), 9-13.

Guderian, P. (2007). Wirksamkeitsanalyse außerschulischer Lernorte - Der Einfluss mehrmaliger Besuche eines Schülerlabors auf die Entwicklung des Interesses an Physik. Dissertation. Berlin: Humboldt-Universität.

Guderian, P., \& Priemer, B. (2008). Interessenförderung durch Schülerlaborbesuche - eine Zusammenfassung der Forschung in Deutschland. Physik und Didaktik in Schule und Hochschule, 2(7), 27-36.

Haucke, K. (2013). Berufsorientierung im Chemieunterricht. Erhebung von Schülervorstellungen zu ausgewählten Berufen und Entwicklung von Konzepten zur Integration von Berufsorientierung in Unterricht und Lehrerbildung. Dissertation. Oldenburg: Carl von Ossietzky Universität.

Haupt, O.J. (2018). Naturwissenschaftlich-technische Umweltbildung in Schülerlaboren: Inventarisierung, Qualitätssicherung und Netzwerkbildung mit Dissemination von Umweltbildungsangeboten. Dänischenhagen: LernortLabor - Bundesverband der Schülerlabore e.V. Abschlussbericht über ein Netzwerkprojekt gefördert unter dem Az 32152 von der Deutschen Bundesstiftung Umwelt.

Itzek-Greulich, H. (2014). Einbindung des Lernorts Schülerlabor in den naturwissenschaftlichen Unterricht. Empirische Untersuchung zu kognitiven und motivationalen Wirkungen eines naturwissenschaftlichen Lehr-Lernarrangements. Dissertation. Tübingen: Eberhard Karls Universität.

Itzek-Greulich, H., \& Vollmer, C. (2017). Emotional and motivational outcomes of lab work in the secondary intermediate track. The 
contribution of a science center outreach lab. Journal of Research in Science Teaching, 54(1), 3-28.

Katz, I., \& Assor, A. (2007). When choice motivates and when it does not. Educational Psychology Review, 19(4), 429-442.

Klees, G., \& Tillmann, A. (2015). Design-Based Research als Forschungsansatz in der Fachdidaktik Biologie. Journal für Didaktik der Biowissenschaften, 6(1), 91-110.

Köller, O., Trautwein, U., Lüdtke, O., \& Baumert, J. (2006). Zum Zusammenspiel von schulischer Leistung, Selbstkonzept und Interesse in der gymnasialen Oberstufe. Zeitschrift für Pädagogische Psychologie, 20(1), 27-39.

LernortLabor (2017). Bildung für nachhaltige Entwicklung in Schülerlaboren. Berlin: WIRmachenDruck.

LernortLabor (2020). Schülerlabor-Atlas. https://www.schuelerlaboratlas.de/. Zugegriffen: 27. Aug. 2020.

Müller, F. H., Hanfstingl, B., \& Andreitz, I. (2007). Skalen zur motivationalen Regulation beim Lernen von Schülerinnen und Schülern: Adaptierte und ergänzte Version des Academic Self-Regulation Questionnaire (SRQ-A) nach Ryan \& Connell. Wissenschaftliche Beiträge aus dem Institut für Unterrichts- und Schule. Klagenfurt: Alpen-Adria-Universität.

Nickolaus, R., Steffensky, M., \& Parchmann, I. (2018). Expertise zu Effekten zentraler außerschulischer MINT-Angebote. Erstellt im Auftrag des Nationalen MINT Forum e. V.. Stuttgart, Kiel: Nationales MINT Forum. https://www.nationalesmintforum.de/ fileadmin/medienablage/content/veranstaltungen/6_NMG_2018/ pdf/NMF-Expertise_zu_Effekten_zentraler_au_erschulischer_ MINT-Angebote_2018.pdf. Zugegriffen: 27. Aug. 2020.

Orion, N., \& Hofstein, A. (1994). Factors that influence learning during a scientific field trip in a natural environment. Journal of Research in Science Teaching, 31(10), 1097-1119.

Pawek, C. (2009). Schülerlabore als interessefördernde außerschulische Lernumgebungen für Schülerinnen und Schüler aus der Mittel- und Oberstufe. Dissertation. Kiel: Christian-Albrechts-Universität.

Prenzel, M., Artelt, C., Baumert, J., Blum, W., Hammann, M., Klieme, E., \& Pekrun, R. (2008). PISA 2006 in Deutschland. Die Kompetenzen der Jugendlichen im dritten Ländervergleich. Münster: Waxmann.

Rat für Kulturelle Bildung (2019). Jugend / YouTube / Kulturelle Bildung. Horizont 2019. Essen. https://www.rat-kulturelle-bildung.
de/fileadmin/user_upload/pdf/Studie_YouTube_Webversion_ final.pdf. Zugegriffen: 27. Aug. 2020.

Reimann, M., Herzog, S., Parchmann, I., \& Schwarzer, S. (2020). Nanotechnologie in Schülerlabor und Schule: Experimenteller Zugang zu alltagsnahen nanotechnologischen Facetten. Chemie konkret: CHEMKON; Forum für Unterricht und Didaktik, 27(5), 215-223.

Reiss, M. J., \& Mujtaba, T. (2017). Should we embed careers education in STEM lessons? The Curriculum Journal, 28(1), 137-150.

Rheinberg, F. (1996). Von der Lernmotivation zur Lernleistung: Das liegt dazwischen. In J. Möller \& O. Köller (Hrsg.), Emotionen, Kognitionen und Schulleistung (S. 23-52). Weinheim: PVU.

Ryan, R. M., \& Deci, E. L. (2000). Intrinsic and extrinsic motivations. Classic definitions and new directions. Contemporary Educational Psychology, 25(1), 54-67.

Sasson, I. (2019). Participation in research apprenticeship program. Issues related to career choice in STEM. International Journal of Science and Mathematics Education, 17(3), 467-482.

Schwarzer, S., \& Itzek-Greulich, H. (2015). Möglichkeiten und Wirkungen von Schülerlaboren. Vor- und Nachbereitung zur Vernetzung mit dem Schulunterricht. Naturwissenschaften im Unterricht: Chemie, 26(147), 8-13.

Schwarzer, R. \& Jerusalem, M. (2002). Das Konzept der Selbstwirksamkeit. Zeitschrift für Pädagogik, Beiheft 44, 28-53.

Stocklmayer, S. M., Rennie, L. J., \& Gilbert, J. K. (2010). The roles of the formal and informal sectors in the provision of effective science education. Studies in Science Education, 46(1), 1-44.

Taskinen, P.H., Schütte, K., \& Prenzel, M. (2013). Adolescents' motivation to select an academic science-related career. The role of school factors, individual interest, and science self-concept. $E d u$ cational Research and Evaluation, 19(8), 717-733.

Weßnigk, S. (2013). Kooperatives Arbeiten an industrienahen außerschulischen Lernorten. Dissertation. Kiel: Christian-AlbrechtsUniversität.

Wilde, M., \& Bätz, K. (2006). Einfluss unterrichtlicher Vorbereitung auf das Lernen im Naturkundemuseum. Zeitschrift für die Didaktik der Naturwissenschaften, 12, 77-88.

Wirth, R. (2019). Berufsorientierung im außerschulischen Lernort mit chemiebezogenen Berufen im Umweltschutz. Ergebnisse einer Fragebogenstudie mit Schülerinnen und Schülern der Sekundarstufe I. Dissertation. Oldenburg: Carl von Ossietzky Universität. 